\title{
DEVELOPMENT AND VALIDATION OF A MODIFIED POLARIMETRIC ASSAY METHOD FOR SMALL VOLUME SAMPLES
}

\author{
K. C. AJITHKUMAR, K. PRAMOD* \\ College of Pharmaceutical Sciences, Govt. Medical College, Kozhikode 673008, Kerala, India \\ Email: pramodkphd@yahoo.com
}

Received: 08 Sep 2017, Revised and Accepted: 10 Oct 2017

\begin{abstract}
Objective: The objective of the present work was to develop a method to be useful for analyzing small volume samples of an optically active compound.

Methods: The method employed quartz cuvette instead of a polarimeter tube. Quantification of optically active compound was successfully validated in terms of linearity and range, precision and robustness. The detection limit (DL) and quantitation limit (QL) were determined as per the ICH guidelines.

Results: Polarimetry generally involves the quantification of optically active compounds by measuring its ability to rotate (in terms of angle of rotation) polarized light passed through it. The plot of angle of rotation versus concentration was linear in wide range of concentration with excellent coefficient of determination of $0.9989 \pm 0.0008$. The low values of $\%$ relative standard deviation (RSD) for repeatability and intermediate precision suggested an excellent precision of the developed polarimetric method. The detection limit (DL) and quantitation limit (QL) were
\end{abstract} determined were found to be 0.5025 and $1.5225 \% \mathrm{w} / \mathrm{v}$ respectively.

Conclusion: A modified polarimetric method for the quantification of dextrose was successfully developed and validated. The method employed quartz cuvette instead of polarimeter tube. The method is expected to be useful in a variety of industries where dextrose finds its application. The method can also be extended to other optically active compounds.

Keywords: ICH, Linearity, Optical activity, Range, Precision, Robustness, Detection limit, Quantitation limit

(C) 2017 The Authors. Published by Innovare Academic Sciences Pvt Ltd. This is an open access article under the CC BY license (http://creativecommons.org/licenses/by/4.0/) DOI: http://dx.doi.org/10.22159/ijap.2017v9i6.22459

\section{INTRODUCTION}

Polarimetry generally involves the quantification of optically active compounds by measuring its ability to rotate (in terms of angle of rotation) polarized light passed through it. A reasonable number of papers have been published on polarimetric assay in drug formulations [1], in vivo studies [2] and biochemistry [3]. But till date, no studies have been reported a validated polarimetric assay employing small sample volumes for quantification. Towards this objective of quantification of optically active compounds by polarimetric assay in a small volume of samples, efforts have been made towards the development and validation of an analytical method by polarimetry. In the present study, dextrose was used as the optically active compound.

\section{MATERIALS AND METHODS}

\section{Materials}

Anhydrous dextrose was purchased from Medilise Chemicals, Kannur, India. Reagent grade I water (Millipore, Molsheim, France) was used for the study.

\section{Polarimetry}

We employed SAC-is accharimeter (Atago Co. Ltd, Tokyo, Japan) in the study. The method employed quartz cuvette as the sample holder. The method was validated according to ICH guidelines, Q2(R1) [4] with respect to linearity and range, precision, robustness, detection limit (DL) and quantitation limit (QL). The angle of rotation of the solutions was determined at a wavelength of $589 \mathrm{~nm}$ and $25^{\circ} \mathrm{C}$.

Fig. 1 displays the photographs describing the modified polarimetric assay method for small volume samples. Our method employed quartz cuvette (used for UV-Vis spectrophotometry) instead of conventional polarimetry tube. Conventional polarimetry tubes have several disadvantages such as high cost, the requirement of large sample volume and need of bubble trap. In this scenario, quartz cuvettes are advantages in terms of low cost (in comparison to conventional polarimetry tube) and requirement of small sample volumes (around $1 \mathrm{ml}$ is sufficient). Moreover, there is no need for bubble trap in the case of quartz cuvette.
Quartz does not absorb at $589 \mathrm{~nm}$ (the sodium D line) employed in the study and therefore is well suited for this purpose. A fixed path length of $1 \mathrm{~cm}$ is an added advantage of quartz cuvette.

\section{Preparation of standard solutions}

Anhydrous dextrose $(25 \mathrm{~g})$ was dissolved in water in a $100 \mathrm{ml}$ volumetric flask and then the volume was made up with water. The dilutions of this stock solution were made by diluting the required aliquot with water to obtain a standard solution in the range of 2-25 $\% \mathrm{w} / \mathrm{v}$. The angle of rotation of the resultant solutions was determined at a wavelength of $589 \mathrm{~nm}$ and $25^{\circ} \mathrm{C}$.

\section{Linearity and range}

The calibration curve was plotted using the concentration range of $2-25 \% \mathrm{w} / \mathrm{v}$. The angle of rotation of the solutions was determined at a wavelength of $589 \mathrm{~nm}$ and $25{ }^{\circ} \mathrm{C}$. A calibration curve was constructed by plotting angle of rotation versus concentration of the standard solution and the regression equation was determined. The experiment was carried out in triplicate.

\section{Precision}

Three concentrations of dextrose solution $(2,8$ and $20 \% \mathrm{w} / \mathrm{v})$ were prepared. The precision of the method was assessed by analyzing dextrose for repeatability and intermediate precision.

\section{(a) Repeatability}

Repeatability (intra-day) was assessed by analyzing dextrose in three different concentrations $(2,8$ and $20 \% \mathrm{w} / \mathrm{v})$ of three times a day. The $\%$ relative standard deviation (RSD) was calculated for the angle of rotation thus obtained, to obtain the intra-day variation.

\section{(b) Intermediate precision}

Intermediate precision (inter-day) was established by analyzing three different concentrations $(2,8$ and $20 \% \mathrm{w} / \mathrm{v})$ of dextrose for three different days. The $\%$ relative standard deviation (RSD) was calculated for the angle of rotation thus obtained, to obtain the inter-day variation. 


\section{Robustness}

The effect of different positions of placement of cuvette with the sample in the polarimeter was assessed by analyzing dextrose in three different concentrations $(2,8$ and $20 \% \mathrm{w} / \mathrm{v})$ by placing at three positions viz. extreme left, center and extreme right in the path of light. The \% RSD was calculated for the angle of rotation thus obtained, to obtain the intra-day variation.

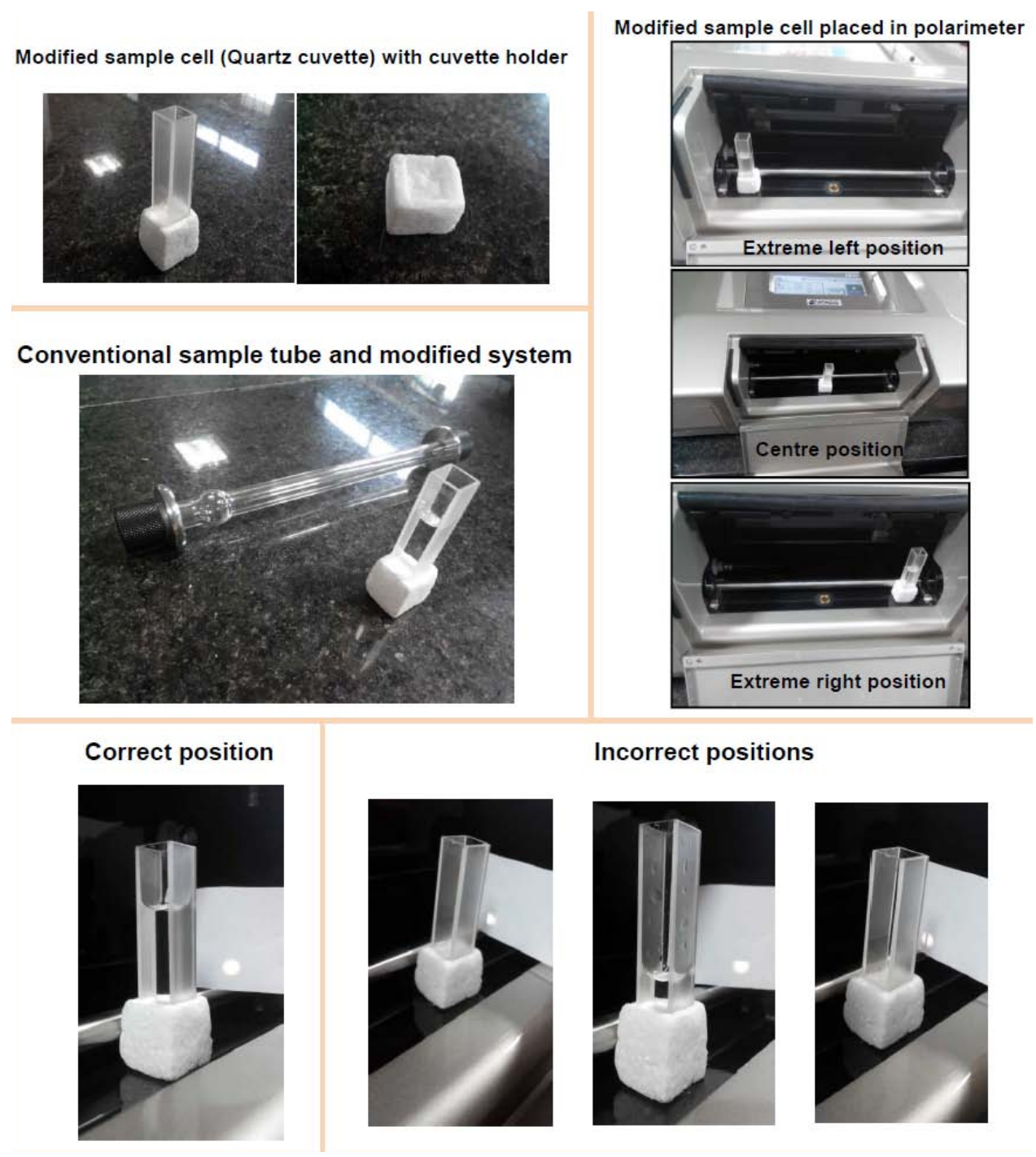

Fig. 1: Photographs of modified instrumentation of polarimetric assay method for small volume samples

\section{Detection and quantitation limits}

The limit of quantification and limit of detection were determined according to equations (1) and (2).

$$
\begin{aligned}
Q L & =10 \sigma / S \\
\mathrm{DL} & =3.3 \sigma / \mathrm{S}
\end{aligned}
$$

where $\sigma$ is the standard deviation of the intercept of the calibration plot and $\mathrm{S}$ is the slope of the calibration curve.

\section{RESULTS AND DISCUSSION}

The modified polarimetric method for the estimation of dextrose in was developed and validated.

\section{Linearity and range}

The angle of rotation of the prepared standard solutions (2-25 $\% \mathrm{w} / \mathrm{v}$ ) was determined. The mean angle of rotation was found to be $0.102-1.277$. The plot of the angle of rotation versus concentration was linear in above concentration range $2-25 \% \mathrm{w} / \mathrm{v}$ with excellent coefficient of determination of $0.9989 \pm 0.0008$. The calibration curve data for dextrose in wateris displayed in table 1 and the calibration plot is displayed in fig. 2 .

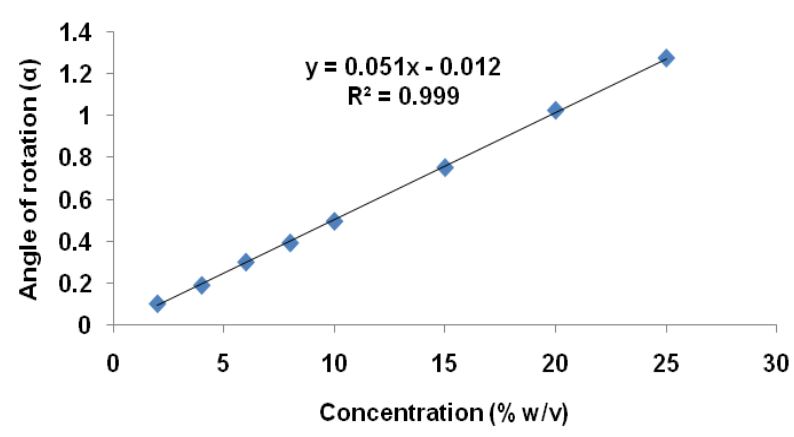

Fig. 2: Calibration plot of dextrose in water 
Table 1: Calibration curve data for dextrose in water

\begin{tabular}{lll}
\hline Concentration $(\% \mathbf{w} / \mathbf{v})$ & Angle of rotation $(\boldsymbol{\alpha})$ & Regressed angle of rotation $^{\mathbf{R}^{2}}$ \\
\hline 2 & $0.102 \pm 0.003$ & 0.0908 \\
4 & $0.190 \pm 0.009$ & 0.1939 \\
6 & $0.301 \pm 0.009$ & 0.2969 \\
8 & $0.393 \pm 0.018$ & 0.3999 \\
10 & $0.496 \pm 0.009$ & 0.5030 \\
15 & $0.753 \pm 0.031$ & 0.7607 \\
20 & $1.027 \pm 0.012$ & 1.0183 \\
25 & $1.277 \pm 0.015$ & 1.2760 \\
\hline
\end{tabular}

Values represent mean $\pm S D, n=3, R^{2}$ : Regression coefficient

\section{Precision}

The precision of the method was assessed by analyzing three different concentrations $(2,8$ and $20 \% \mathrm{w} / \mathrm{v})$ of dextrose.

\section{(a) Repeatability}

Repeatability (intra-day) was assessed by analyzing three different concentrations $(2,8$ and $20 \% \mathrm{w} / \mathrm{v}$ ) of dextrose three times a day. The $\%$ RSD was calculated for the angle of rotation thus obtained, to obtain the intra-day variation and is given in table 2 .

\section{(b) Intermediate precision}

Intermediate precision (inter-day) was established by analyzing three different concentrations $(2,8$ and $20 \% \mathrm{w} / \mathrm{v}$ ) of dextrose for three different days. The \% RSD was calculated, to obtain the interday variation and is given in table 2 .
The low values of \% RSD for repeatability and intermediate precision suggested an excellent precision of the developed polarimetric method.

\section{Robustness}

The results of the study for evaluation of robustness in the positioning of the sample during the polarimetric quantification method are displayed in table 3. The low values of $\%$ RSD for the angle of rotation indicated the excellent robustness of the developed polarimetric method.

\section{Detection and quantitation limits}

The detection limit (DL) and quantitation limit (QL) were determined as per the ICH guidelines and were found to be 0.5025 and $1.5225 \% \mathrm{w} / \mathrm{v}$ respectively.

Table 4 summarizes the linear regression and validation data of polarimetric method for the quantification of dextrose in water.

Table 2: Repeatability and intermediate precision

\begin{tabular}{lllll}
\hline Concentration (\% w/v) & Repeatability & & Intermediate precision & \% RSD \\
\cline { 2 - 5 } & Angle of rotation $(\boldsymbol{\alpha})$ & \% RSD & Angle of rotation $(\boldsymbol{\alpha})$ & 0.96 \\
\hline 2 & $0.108 \pm 0.004$ & 3.70 & $0.104 \pm 0.001$ & 1.49 \\
8 & $0.404 \pm 0.015$ & 3.71 & $0.403 \pm 0.006$ & 2.11 \\
20 & $1.001 \pm 0.008$ & 0.80 & $0.995 \pm 0.021$ & \\
\hline
\end{tabular}

Values represent mean $\pm \mathrm{SD}, \mathrm{n}=3, \mathrm{RSD}=$ Relative standard deviation

Table 3: Data for robustness of polarimetric method

\begin{tabular}{lllll}
\hline Concentration (\% w/v) & Position of cuvette & & \multicolumn{2}{c}{ Angle of rotation ( $\boldsymbol{\alpha})$} \\
\cline { 2 - 4 } & Extreme left & Centre & Extreme right & \\
\hline 2 & 0.094 & 0.092 & 0.090 & $0.092 \pm 0.002$ \\
8 & 0.382 & 0.384 & 0.381 & $0.382 \pm 0.002$ \\
20 & 0.969 & 0.971 & 0.969 & $0.970 \pm 0.001$ \\
\hline
\end{tabular}

Values represent mean $\pm S D, n=3, R S D=$ Relative standard deviation

Table 4: Linear regression and validation data

\begin{tabular}{ll}
\hline Parameter & Data \\
\hline Regressionanalysis & \\
Slope & $0.05153 \pm 0.00055$ \\
Intercept & $-0.01226 \pm 0.00785$ \\
Regression coefficient $\left(\mathrm{R}^{2}\right)$ & $0.9989 \pm 0.0008$ \\
Validation & \\
Range $(\% \mathrm{w} / \mathrm{v})$ & $2-25$ \\
Detection limit (DL) (\%w/v) & 0.5025 \\
Quantitation limit (QL) $(\% \mathrm{w} / \mathrm{v})$ & 1.5225 \\
\hline
\end{tabular}

Values represent mean $\pm \mathrm{SD}, \mathrm{n}=3$

\section{CONCLUSION}

A modified polarimetric method for the quantification of dextrose was successfully developed and validated. The method employed quartz cuvette instead of polarimeter tube. Quartz does not absorb at $589 \mathrm{~nm}$ and therefore is well suited for this purpose. Cost of quartz cuvette is very less compared to conventional polarimeter sample tubes. There is no need for bubble trap in the case of quartz cuvette. The modified method was validated in terms of linearity and range, precision and 
robustness. The detection limit (DL) and quantitation limit (QL) were determined as per the ICH guidelines and were found to be 0.5025 and $1.5225 \% \mathrm{w} / \mathrm{v}$ respectively.

The method is expected to be useful in a variety of industries where dextrose finds its application. The method can also be extended to other optically active compounds.

\section{ACKNOWLEDGEMENT}

K. C. Ajithkumar gratefully acknowledges Kerala State Council for Science, Technology and Environment (KSCSTE), Thiruvananthapuram, India, for sanctioning student project (No. 14/SPS 57/2016/KSCSTE, Dated 02.04.2016).

\section{CONFLICT OF INTERESTS}

Declared none

\section{REFERENCES}

1. Yuan A, De-jiang S. Polarimetric assay of netilmicin sulfate eye drops. Chin J Pharm Anal 2005;25:986-7.

2. Purvinis G, Cameron BD, Altrogge DM. Noninvasive polarimetric-based glucose monitoring: an in vivo study. J Diabetes Sci Technol 2011;5:380-7.

3. Blass DA, Adams E. Polarimetry as a general method for enzyme assays. Anal Biochem 1976;71:405-14.

4. ICH-Guidelines Q2 (R1). Validation of analytical procedures: text and methodology. Geneva, Switzerland: 2005. 